\title{
Two-step ESD: an option for en-bloc resection of extensive colorectal laterally spreading tumors
}

\section{(ㄷ)(1)}

Authors

Fabio S. Kawaguti ${ }^{1}$, Ossamu Okazaki ${ }^{1}$, Nelson T. Miyajima ${ }^{2}$, Vanderlei Segateli ${ }^{1}$, Carlos F.S. Marques ${ }^{1}$, Caio S.R. Nahas $^{1}$, Bruno C. Martins ${ }^{1}$, Sergio C. Nahas ${ }^{1}$, Ulysses R. Junior ${ }^{1}$, Fauze M. Filho ${ }^{1}$

Institutions

1 Cancer Institute of São Paulo University Medical School, São Paulo, Brazil

2 Gastrointestinal Endoscopy Unit, Hospital das Clinicas, University of São Paulo Medical School, São Paulo, Brazil

submitted 26.10.2018

accepted after revision 14.2.2019

Bibliography

DOI https://doi.org/10.1055/a-0887-4294 |

Endoscopy International Open 2019; 07: E1092-E1096

(c) Georg Thieme Verlag KG Stuttgart · New York elSSN 2196-9736

Corresponding author

Fabio Shiguehissa Kawaguti, MD, Cancer Institute of University of Sao Paulo, Dr. Arnaldo Av, 251, 01246-903,

São Paulo, Brazil

Fax: +55-11-3069-7579

shiguehiss@yahoo.com.br

\section{ABSTRACT}

Background and study aims Endoscopic submucosal dissection (ESD) is considered feasible and safe for treatment of colorectal laterally spreading tumors (LST), However it remains a challenge in case of extensive lesions even for experts. This study aimed to describe a new method to facilitate ESD of extensive colorectal LSTs. Between July 2010 and January 2018, 140 patients underwent ESD for colorectal LSTs. Four of them were submitted to two-step ESD and were included in this retrospective study. The submucosal dissection of lesions larger than $12 \mathrm{~cm}$ started and continued until the medical team decided to pause the procedure and continue it in a second step. The second procedure was performed 2 days after to finish the en-bloc resection. Three patients were male, with mean age of $67.2 y( \pm 2.2)$. All lesions were located in the rectum, with a mean size of $153.7 \mathrm{~mm}( \pm 33.8)$. En-bloc and curative resection were successfully achieved in all cases. Mean duration of the first step of the procedure was 255 minutes $( \pm 61.8)$, and mean duration of the second step was 205 minutes $( \pm 205)$. Overall mean duration of both steps was 460 minutes $( \pm 168)$. Mean dissected area in the first step of the procedure was approximately $55 \%$ of the lesion. No adverse events were observed. In conclusion, our results suggest that performing ESD in two steps could be a feasible and safe option for exceptional cases in which is not possible to finish the procedure in one step, avoiding the morbidity of surgical treatment.

\section{Introduction}

Endoscopic mucosal resection (EMR) is a well-established treatment for colorectal laterally spreading tumor (LTS) [1]. However, for lesions over $20 \mathrm{~mm}$, the probability of piecemeal resection and local recurrence are higher [2].

Endoscopic submucosal dissection (ESD), initially developed for treatment of gastric neoplasms, made possible complete en-bloc resection of colorectal LST larger than $20 \mathrm{~mm}$ [3]. Currently, ESD is feasible and safely performed in Asian countries and in some western centers [4].
For extensive lesions, ie, those measuring over $10 \mathrm{~cm}$, ESD is associated with technical difficulty, longer procedural time, and higher risk of adverse events [5].

There are only a few case reports of en-bloc resection with ESD of extensive LSTs. Even for experts, under ideal circumstances and with adequate accessories, performing the procedure in these cases remains a challenge [5]. Therefore, the aim of this study was to describe an alternative that enables en-bloc ESD of extensive LSTs: two-step ESD. 

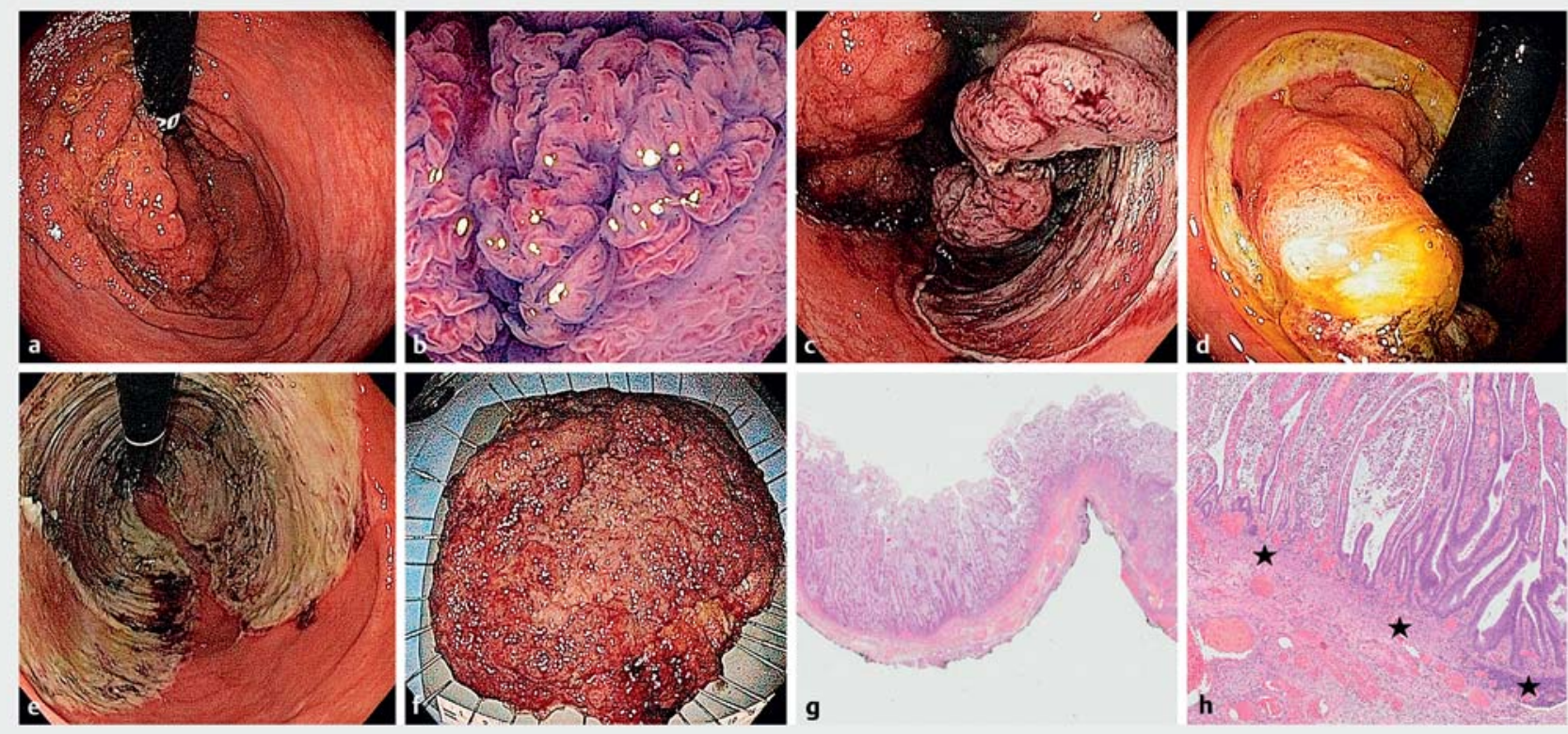

- Fig. 1 a Extensive lateral-spreading tumor at distal rectum. b Magnifying colonoscopy combined with indigo carmine dye with Vi pit pattern. c ESD - end of first procedure. $\mathbf{d}$ ESD - start of second procedure. Fibrin layer in the previous dissected area. e Final aspect after two steps ESD. f Stretched 150-mm specimen showing ischemic areas corresponding to the first procedure. $\mathbf{g}$ Tubulo-villous adenoma with ischemic changes, with partial necrosis of the mucosa. Edema and vascular congestion in the submucosa. The marked area is shown in $\mathbf{h}(\mathrm{HE}, 0,4 \times)$. $\mathbf{h}$ Ischemic changes in tubulo-villous adenoma. Mucosa with total necrosis (left side) or partial necrosis (right). There is preservation of the intestinal wall layering architecture, with a clear view of muscularis of mucosa (stars). (HE, $4 \times$ ).

\section{Case reports}

Between July 2010 and January 2018, 140 patients underwent ESD for colorectal LSTs at Cancer Institute of University of São Paulo. Among them, 21 patients had colorectal LSTs larger than $10 \mathrm{~cm}$ and four of them underwent two-step ESD, all of whom had LSTs larger than $12 \mathrm{~cm}$. Data from a prospectively collected database were analyzed retrospectively with respect to en-bloc resection rate, procedural duration, adverse events (AEs), histology, and length of hospital stay.

All patients underwent colonoscopy with magnification and the lesions were classified according to Kudo pit pattern classification to confirm absence of massive submucosal (SM) invasion [6]. Lesions with noninvasive pattern were considered eligible for ESD.

\section{Procedure}

Inpatient bowel preparation was performed with $20 \mathrm{mg}$ of bisacodyl 1 day before the procedure, and a $10 \%$ mannitol solution on the morning of the procedure at the endoscopy division. In our experience, bowel preparation during the night shift presents unsatisfactory results. Therefore, the ESD procedures were scheduled for the afternoon after adequate fasting.

The procedures were performed by ESD expert endoscopists (FSK, NTM), under general anesthesia. A forward-viewing endoscope (GIF-H180; Olympus) was used with a transparent distal hood attachment (D201-10704; Olympus). Carbon dioxide was used for insufflation. After submucosal injection of a mannitol $10 \%$ solution with indigo carmine and appropriate mucosal lift- ing, precutting of mucosa followed by ESD was performed with a flush knife (DK2618LN; Fujifilm Medical, Tokyo, Japan).

Injection was repeated as needed and ESD of the lesions continued until the medical team decided to pause the procedure and continue it a second time.

Among the reasons considered for pausing the procedure was the logistical issue of the unit, which is an outpatient endoscopy unit, with structure to operate until $7 \mathrm{pm}$. For cases in which en bloc lesion resection could not be completed before that time, a two-step ESD was planned. Other aspects evaluated were fatigue of the endoscopist and assistant team in addition to prolonged anesthesia time. All cases were paused until 5:30 pm to ensure extubation and patient referral to ward before $7 \mathrm{pm}$. The dissected part of the lesion was left in situ and the procedure was scheduled to continue 2 days later.

In the morning and afternoon of the day after the first procedure, a clear liquid diet was offered, followed by a night bowel preparation.

During the second step of the procedure, signs of ischemia in the dissected flap were identified with a thick layer of fibrin covering the resection bed, hindering resumption of dissection. No necrosis or greater friability of the muscular layer was identified, which could increase risk of perforation. The lesion limits remained clearly identifiable.

The key point for successful ESD at second procedure was correct identification of the previous submucosal plane. A submucosal injection was used to lift the mucosa of the remaining lesion and clear the fibrin layer that covered the resection bed, revealing the submucosal plane of the dissection. After ade- 
- Table 1 Clinical characteristics of the patients and lesions.

\begin{tabular}{|c|c|c|c|c|c|c|c|c|c|c|c|}
\hline $\begin{array}{l}\text { Pa- } \\
\text { tient }\end{array}$ & $\begin{array}{l}\text { Age } \\
\text { (years) }\end{array}$ & Sex & $\mathrm{CCl}$ & ASA & $\begin{array}{l}\text { KAR- } \\
\text { NOFSKY }\end{array}$ & Site & $\begin{array}{l}\text { Lesion } \\
\text { size } \\
(\mathrm{mm})\end{array}$ & $\begin{array}{l}\text { Circunferetial } \\
\text { extension (\%) }\end{array}$ & $\begin{array}{l}\text { Extent to } \\
\text { dentate } \\
\text { line }\end{array}$ & $\begin{array}{l}\text { Macro- } \\
\text { scopic } \\
\text { type }\end{array}$ & $\begin{array}{l}\text { Pit } \\
\text { pattern }\end{array}$ \\
\hline 1 & 70 & $M$ & 2 & II & 100 & Rectum & 150 & $\geq 90 \%<100 \%$ & Yes & LST-GM & $\mathrm{Vi}$ \\
\hline 2 & 68 & $\mathrm{M}$ & 3 & II & 100 & Rectum & 125 & $\geq 90 \%<100 \%$ & No & LST-GM & Vi \\
\hline 3 & 64 & $\mathrm{~F}$ & 2 & II & 100 & Rectum & 210 & $100 \%$ & Yes & LST-GH & Vi \\
\hline 4 & 67 & $M$ & 3 & III & 90 & Rectum & 130 & $\geq 90 \%<100 \%$ & Yes & LST-GM & $\mathrm{Vi}$ \\
\hline
\end{tabular}

- Table 2 Results of two-step ESD.

\begin{tabular}{|l|c|}
\hline 1st procedure mean duration (min) & $255( \pm 61.8)$ \\
\hline 2nd procedure mean duration (min) & $205( \pm 115.2)$ \\
\hline Total procedure mean duration (min) & $460( \pm 168.3)$ \\
\hline 1st procedure mean dissected area (\%) & $55( \pm 17.3)$ \\
\hline Mean length of hospital stay (days) & $4.7(4-5)$ \\
\hline Mean follow-up (days) & $266.2( \pm 255.4)$ \\
\hline En-bloc resection rate (\%) & 100 \\
\hline Curative resection rate (\%) & 100 \\
\hline Imediate bleeding rate (\%) & 0 \\
\hline Delayed bleeding rate (\%) & 0 \\
\hline Perforation rate (\%) & 0 \\
\hline Post-ESD stenosis rate (\%) & 50 \\
\hline ESD, endoscopic submucosal dissection & \\
\hline
\end{tabular}

quate identification of the submucosal plane, there was no difficulty in finishing the resection.

The specimens were fixed on a corkboard with pins and immersed into $1 \%$ formalin solution. The pathologist was asked to evaluate for signs of ischemia in the resected specimen.

One month after ESD, a rectoscopy was performed to check for stenosis. If present, it was treated with either digital or balloon dilatation sessions. In the absence of stenosis, surveillance endoscopic examination was planned to check for recurrence 6 months after ESD and every year thereafter.

A single pathologist, experienced in ESD resected specimens, assessed all the lesions.

Four patients with extensive rectal LSTs were treated with two-step ESD. Three were male, with mean age of 67.2 y $( \pm 2.2)$.

One lesion was circumferential ( $\triangleright$ Fig. 1 ), and three lesions involved $\geq 90 \%$ and $\leq 100 \%$ of the circumference of the rectum, with a mean size of $153.7 \mathrm{~mm}( \pm 33.8)$. Three lesions were macroscopically classified as granular mixed-type LST and one as granular homogeneous-type LST. Magnification colonoscopy showed a noninvasive pattern in all lesions. The characteristics of the patients and lesions are shown in $>$ Table 1.

Mean procedure duration was 255 minutes ( \pm 61.8$)$ and 205 minutes $( \pm 205)$ for the first and second step, respectively. The overall mean duration was 460 minutes $( \pm 168)$. The mean dissected area in the first procedure was approximately $55 \%$ of the lesion.

En-bloc resection was successfully achieved in all cases, and there were no cases of early or delayed bleeding or perforation. Two patients developed post-ESD stenosis, both of which were successfully treated, one with balloon dilation sessions and one with digital dilation. Mean length of hospital stay was 4.75 days (4-5 days). Mean time of follow-up was 266.25 days ( \pm 255.4 ). Results are summarized in $>$ Table 2 . Representative images of the cases are shown in > Fig. 1 and $>$ Fig. 2.

Regarding histology, three lesions were well-differentiated intramucosal adenocarcinoma in a tubulovillous adenoma and the other was a traditional serrated adenoma with high-grade dysplasia. There was no evidence of lymphovascular invasion in the resected specimens. $\mathrm{R} 0$ en-bloc resection, was achieved in all cases (100\%). The pathologist reported limited ischemic necrosis in the area resected during the first step of the procedure in all patients. The necrosis, however, was restricted to a limited area of the resected specimen and did not impact histopathological assessment ( $\triangleright$ Fig. 2).

\section{Discussion}

In our institution, three of 21 patients with extensive colorectal LSTs had unsuccessful endoscopic resection attempts, of whom two were referred for surgical resection and one for complementary piecemeal EMR resection. All patients in whom ESD failed had lesions larger than $15 \mathrm{~cm}$ and a prolonged procedure time.

It is recognized that a prolonged procedure time leads to stress and fatigue of the surgeon, reducing his/her effectiveness, increasing risk of medical error, and even contributing to failure of surgical procedures [7].

Thus, to minimize failure of endoscopic resection, it was decided to plan ESD in two steps in cases of extensive lesions with a foreseen prolonged procedural time and in cases in 


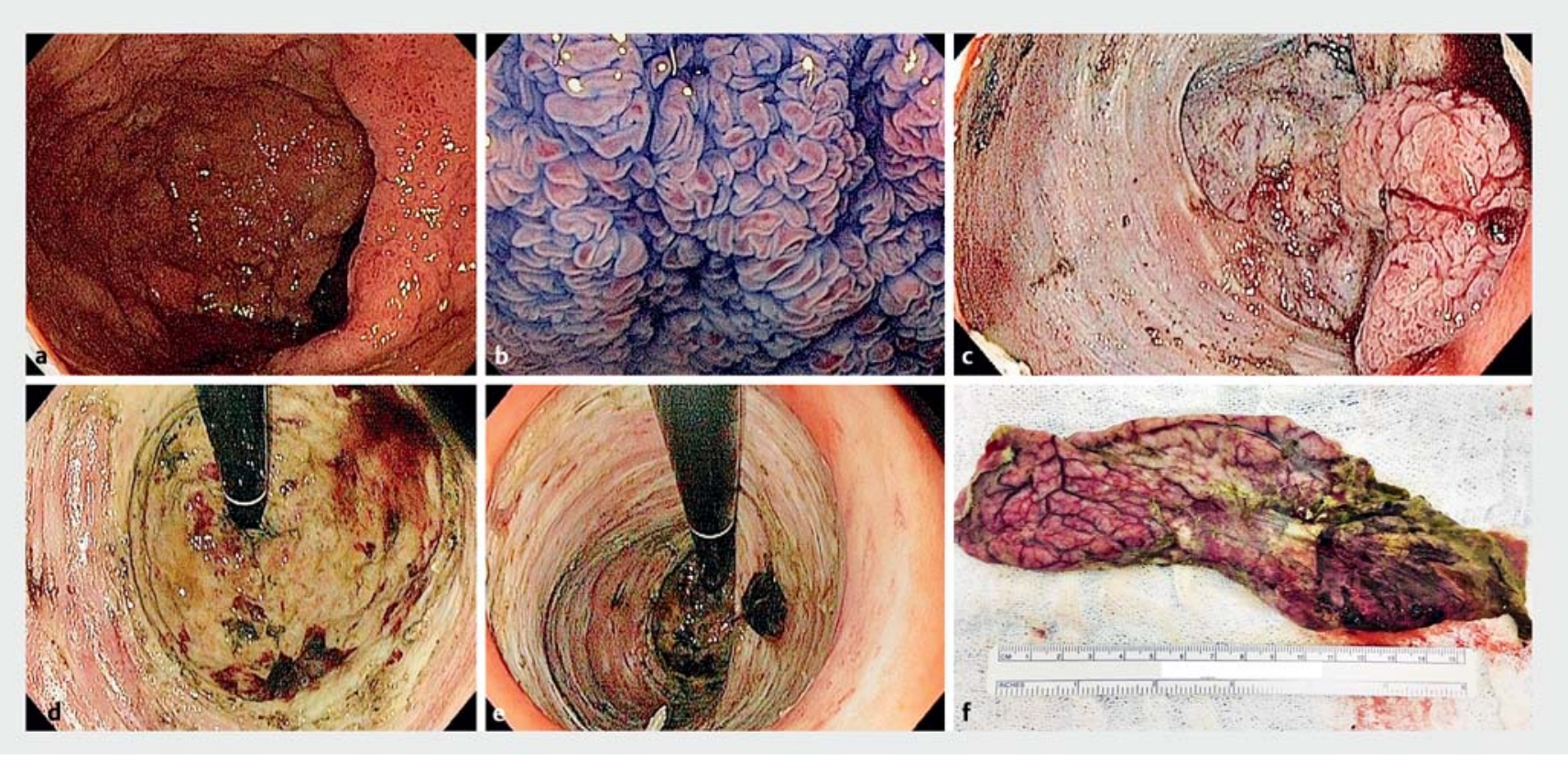

- Fig. 2 a Extensive circumferential lateral spreading tumor at rectum. b Magnifying colonoscopy combined with indigo carmine dye. c ESD end of first procedure. $\mathbf{d}$ ESD - second procedure. Resection bed with clear differentiation of dissected areas in the first (yellowish areas with fibrin) and second procedure. e Final aspect after two-step ESD. $\mathbf{f}$ En-bloc specimen of circumferential lesion, measuring $210 \mathrm{~mm}$, showing ischemic areas corresponding to the first procedure.

which the procedure time was prolonged such that they could not be finished before the endoscopic unit closed.

Prediction of procedure time can be challenging. Based on our experience, bulky lesions larger than $15 \mathrm{~cm}$ and with extension to dentate line seem to be associated with prolonged procedure time.

In this study, besides large tumor size, intense fibrosis of the submucosal layer, which may lead to poor lifting and has been related with failure of en-bloc resection and perforation, was a factor in two lesions [8]. Moreover, three lesions presented extension to the dentate line, considered a factor predictive of incomplete excision [9]. These factors increase the degree of technical difficulty of ESD, increasing procedural time.

Two-step ESD allowed en-bloc resection of these lesions, with a curative resection rate of $100 \%$ (4/4) and no major related AEs. All patients remain under endoscopic surveillance during a mean follow-up time of 266.2 days, without signs of recurrence.

The high incidence of rectal stenosis (50\%) was expected, given the extension of the dissection involving more than $90 \%$ of the rectal circumference, as previously reported [10]. All patients with stenosis were treated successfully by dilation sessions.

Concerning histology, all lesions were restricted to the mucosal layer. Presence of necrotic tissue and ischemic changes in the flap dissected in the first step of the procedure interfered with but did hinder assessment of the cure criteria on the resected specimen.

Two-step ESD increases the length of hospital stay and adds an additional general anesthesia, with all costs and risks of related AEs. In addition, it may be stressful for patients. How- ever, it allows minimally invasive en-bloc resection in challenging cases, preserving the rectum and avoiding a permanent colostomy. Therefore, for exceptional cases, it might be a useful alternative.

\section{Conclusion}

In conclusion, our results suggest that performing ESD in two steps could be a feasible and safe option for exceptional cases in which is not possible to finish the procedure in one step, avoiding the morbidity of surgical treatment.

\section{Acknowledgements}

The authors would like to acknowledge the contribution of Drs. Shin-ei Kudo and Takemasa Hayashi (Showa University Northern Yokohama Hospital), Takashi Toyonaga (Kobe University), Yutaka Saito (National Cancer Center, Tokyo) and Haruhiro Inoue (Showa University Koto Toyosu Hospital, Tokyo) in the learning process of our staff in the diagnosis and treatment of early gastrointestinal cancer.

\section{Competing interests}

\section{None}

References

[1] Saito $\mathrm{Y}$, Fujii T, Kondo $\mathrm{H}$ et al. Endoscopic treatment for laterally spreading tumors in the colon. Endoscopy 2001; 33: 682-686 
[2] Hotta K, Fujii T, Saito Y et al. Local recurrence after endoscopic resection of colorectal tumors. Int J Colorectal Dis 2009; 24: 225-230

[3] Tanaka S, Toyonaga T, Morita Y. Feasibility and safety of endoscopic submucosal dissection for large colorectal tumors. Surg Laparosc Endosc Percutan Tech 2015; 25: 223-228

[4] Kawaguti FS, Nahas CSR, Marques CFS et al. Endoscopic submucosal dissection versus transanal endoscopic microsurgery for the treatment of early rectal cancer. Surg Endosc 2014; 28: 1173-1179

[5] Jung DH, Youn YH, Kim JH et al. Endoscopic submucosal dissection for colorectal lateral spreading tumors larger than $10 \mathrm{~cm}$ : Is it feasible? Gastrointest Endosc 2015; 81: 614-620

[6] Kudo S, Tamura S, Nakajima T et al. Diagnosis of colorectal tumorous lesions by magnifying endoscopy. Gastrointest Endosc 1996; 44: $8-14$
[7] McCormick F, Kadzielski J, Landrigan CP et al. Surgeon fatigue: a prospective analysis of the incidence, risk, and intervals of predicted fatigue-related impairment in residents. Arch Surg 2012; 147: 430 435

[8] Matsumoto A, Tanaka S, Oba S et al. Outcome of endoscopic submucosal dissection for colorectal tumors accompanied by fibrosis. Scand J Gastroenterol 2010; 45: 1329-1337

[9] Imai K, Hotta K, Yamaguchi Y et al. Safety and efficacy of endoscopic submucosal dissection of rectal tumors extending to the dentate line. Endoscopy 2015; 47: 529-532

[10] Hayashi T, Kudo S, Miyachi $\mathrm{H}$ et al. Management and risk factor of stenosis after endoscopic submucosal dissection for colorectal neoplasms. Gastrointest Endosc 2017; 86: 358 -369 